\title{
Neural activity during sentence processing as reflected in theta, alpha, beta, and gamma oscillations
}

\author{
Nietzsche H.L. Lam, Jan-Mathijs Schoffelen *, Julia Uddén, Annika Hultén ${ }^{1}$, Peter Hagoort*
}

Donders Institute for Brain, Cognition and Behaviour, Centre for Cognitive Neuroimaging, Radboud University Nijmegen, Nijmegen, Netherlands Max Planck Institute for Psycholinguistics, Nijmegen, Netherlands

\section{A R T I C L E I N F O}

\section{Article history:}

Received 29 July 2015

Accepted 3 March 2016

Available online 9 March 2016

\section{Keywords:}

Oscillations

MEG

Sentence processing

Lexical retrieval

Unification

\begin{abstract}
A B S T R A C T
We used magnetoencephalography (MEG) to explore the spatiotemporal dynamics of neural oscillations associated with sentence processing in 102 participants. We quantified changes in oscillatory power as the sentence unfolded, and in response to individual words in the sentence. For words early in a sentence compared to those late in the same sentence, we observed differences in left temporal and frontal areas, and bilateral frontal and right parietal regions for the theta, alpha, and beta frequency bands. The neural response to words in a sentence differed from the response to words in scrambled sentences in left-lateralized theta, alpha, beta, and gamma. The theta band effects suggest that a sentential context facilitates lexical retrieval, and that this facilitation is stronger for words late in the sentence. Effects in the alpha and beta bands may reflect the unification of semantic and syntactic information, and are suggestive of easier unification late in a sentence. The gamma oscillations are indicative of predicting the upcoming word during sentence processing. In conclusion, changes in oscillatory neuronal activity capture aspects of sentence processing. Our results support earlier claims that language (sentence) processing recruits areas distributed across both hemispheres, and extends beyond the classical language regions.
\end{abstract}

(c) 2016 Elsevier Inc. All rights reserved.

\section{Introduction}

How are you reading this sentence? Conceptually, the reader needs to retrieve and understand the meaning of individual lexical items (words), and combine these items to derive an interpretation spanning its entirety. The latter process is referred to as unification (Hagoort, $2003,2005,2013)$. At the neural level, the fast and incremental nature of sentence processing likely involves multiple brain regions. Yet, we know little about how the brain orchestrates sentence processing because the majority of neuroimaging studies on language processing have focused on the single word level. Of the studies that focused on sentence processing, most used syntactic or semantic anomalies as an experimental manipulation. Considering daily language exposure, this questions the ecological validity of such stimuli. Moreover, although the experimental designs were well controlled, these studies related neural responses only to specific critical events within a sentence. Consequently, the processing of each word in a sentence, and how it is affected by an incremental context, has not been studied in detail. In the

\footnotetext{
* Corresponding authors at: Donders Centre for Cognitive Neuroimaging, Kapittelweg 29, 6525 EN, Nijmegen, Netherlands.

E-mail addresses: jan.schoffelen@donders.ru.nl (J.-M. Schoffelen),

p.hagoort@donders.ru.nl (P. Hagoort).

1 Current affiliation: The Department of Neuroscience and Biomedical Engineering, Aalto University, Helsinki, Finland.
}

current study, we address these two aspects of sentence processing using natural sentences. We focused on the modulation of neural oscillations in response to individual words in the context of a sentence, and examined how this modulation changed as the sentence unfolded.

\section{Oscillatory neural activity}

When studying electrophysiological signals, spectral analysis techniques are aimed at quantifying frequency-specific neural activity. These techniques were initially used to study rhythmic activity during visual processing, or low-level motor behavior, and then gained popularity in the 2000s to study higher order cognition, such as language (Hari and Salmelin, 2012). Beyond capturing the transient response to external events, estimates of frequency-specific activity reflect oscillatory neural activity that is not necessarily time- or phase-locked to an event, as opposed to event-related averages. Thus, it may provide a different but complimentary perspective on how the brain orchestrates language (including sentence) processing. Furthermore, this technique allows us to investigate the relationship between aspects of sentence processing and the spatiotemporal dynamics of oscillatory activity.

The power of oscillatory activity has been observed to be modulated in many cognitive tasks. These modulations are typically described as relative decreases (event-related desynchronization, ERD; Pfurtscheller and Aranibar, 1977) or relative increases (event-related sychronization, ERS; Pfurtscheller, 1992). Depending on the frequency band, such power 
changes may indicate either activation or deactivation of a brain region. An ERD in the gamma band $(>40 \mathrm{~Hz})$ reflects a reduction in processing in underlying cortical regions, but would reflect increased processing when observed in the alpha $(8-12 \mathrm{~Hz})$ or beta $(13-30 \mathrm{~Hz})$ bands (Jensen and Mazaheri, 2010; Klimesch et al., 1997; Osipova et al., 2006; Klimesch et al., 2001). For the theta frequencies, however, it is equivocal as to whether an ERD reflects activation or deactivation. Oscillatory neural activity can be productively studied using MEG. This method has good spatial resolution and excellent temporal resolution (in the order of milliseconds), which enables it to capture rapid (tens of milliseconds) changes associated with cognitive processes in the brain.

\section{Studying sentence processing with oscillations}

Both ERS and ERD have been observed in studies concerned with sentence processing, but most studies analyzed the data on the sensor-level which provided poor spatial localization of the effects, and most of them focused on a single word (that produced a grammatical violation) in the sentence (e.g., Bastiaansen et al., 2010; Davidson and Indefrey, 2007). Across these studies, different frequency bands have been associated with different functional explanations. The theta and alpha bands, for example, have been associated with the lexicalsemantic retrieval of words (Bastiaansen et al., 2005; Klimesch et al., 1997). Beta and gamma band ERS has been suggested to reflect unification of the semantic and syntactic information in sentences, respectively (Bastiaansen and Hagoort, 2015; Bastiaansen et al., 2010; Hald et al., 2006). The alpha and beta bands have also been demonstrated to be involved in syntactic processing (Bastiaansen et al., 2010; Davidson and Indefrey, 2007; Kielar et al., 2015). Overall, these results, whilst suggestive, highlight the need for further study of oscillations in sentence processing, specifically to study multiple frequency bands in one dataset, and to understand their relation to each other.

In sentence processing, words are retrieved from memory and combined into an interpretation of the larger phrase, regulated by semantic and grammatical constraints (Hagoort, 2013). A recent fMRI metaanalysis revealed consistent involvement of the left inferior frontal gyrus (BA 45 and BA 47), left middle temporal gyrus (MTG), and left superior temporal gyrus in sentence processing (Hagoort and Indefrey, 2014). The memory, unification, and control (MUC) model of sentence processing also focuses on these areas (Hagoort, 2003, 2005, 2013). It proposes that the left temporal cortex and angular gyrus are implicated in word retrieval from memory, while unification (maintenance and integration of words) involves the left inferior frontal cortex. Furthermore, as the sentence unfolds, a predictive context results from the interaction between these areas, and this context facilitates the processing of upcoming words. Previous oscillatory studies on sentence processing have, to our knowledge, been restricted to the sensor level. It is therefore unclear whether oscillations localize to similar brain regions as found in event-related M/EEG and fMRI studies. The current study, presenting a thorough source-level analysis of oscillatory activity during sentence processing, addresses this shortcoming.

\section{Current study}

In the present study, we investigated oscillatory power changes during sentence processing. We obtained MEG data while participants read sentences. As a control condition, participants also read lists of words (created by scrambling sentences). Our study focused on exploring which neural areas, at which frequencies are involved in sentence processing. We analyzed the spatiotemporal dynamics of the oscillatory activity by using a beamformer in the frequency domain. This allowed us to better quantify the spatial aspects of the effects, and to improve on the previous studies that could only report results at the sensor-level.

To obtain a complete picture of oscillations at various frequencies, we investigated five frequency bands: theta, alpha, beta, low gamma, and high gamma. We chose to divide the gamma band because previous studies have shown a distinction between low gamma (around 30 to $60 \mathrm{~Hz}$ ) and high gamma (above $80 \mathrm{~Hz}$ ); the precise frequencies for low and high gamma differ between cortical regions and the cognitive task (Crone et al., 1998; Dalal et al., 2008; Hauck et al., 2007). Furthermore, previous sentence-processing studies have only shown effects at around $40-60 \mathrm{~Hz}$, and we were interested in whether effects in higher frequencies were also present. In the current study, we investigated two aspects of sentence processing: The word analysis sought to reveal how single words are integrated into a representation of the entire sentence, and the context analysis assessed the effect of the incremental context on single word processing.

\section{Word analysis}

In order to investigate the oscillatory response to single words within the context of a sentence, we analyzed the responses to each of the single words in a sentence and contrasted them to single words in a random order (word list). Sentences have a structured and meaningful context-semantic and syntactic information that goes beyond the level of the individual words. Word lists on the other hand have no structure and only a weak overarching semantic context (because each word list was created by scrambling a sentence). We hypothesized the sentence context to have a facilitatory effect, since the syntactic and semantic information in preceding words constrain the possibilities of the upcoming word both syntactically and semantically. The effect of the context may be observed in memory retrieval, unification, and prediction (Hagoort and Poeppel, 2013). The sentence context should make memory retrieval easier for sentences than word lists, and because previous studies associated theta power with memory retrieval (Bastiaansen et al., 2005; Hagoort, 2013), we expected differences between the conditions in this frequency band. Since the prediction of the upcoming word within a context has been associated with gamma ERS (Wang et al., 2012), we expected to observe this pattern for sentences but not word lists. Finally, the alpha, beta, and gamma bands have been implicated in unification (e.g., Bastiaansen and Hagoort, 2015; Bastiaansen et al., 2010; Hald et al., 2006); therefore, we expected differences between sentences and word lists in one or more of these frequencies.

\section{Context analysis}

To analyze how the unfolding sentence context affects oscillatory activity over time we quantified the modulation of the oscillatory response between words at early and late positions in a sentence. This provides a view of the long-term changes in power as the sentence progresses. As the sentence unfolds, memory should become taxed because more words need to be retained for unification. As ERS in the theta band has been associated with memory maintenance, we expected an increase in theta power for words in late versus early positions in the sentence. An incremental context can also impose more constraints on how words are being combined, which could facilitate unification as the sentence unfolds. Changes in power across a sentence but not a word list have previously been associated with the beta and gamma bands (Bastiaansen et al., 2010; Hald et al., 2006). Effects in these frequency bands are suggested to reflect unification. Therefore, we hypothesized that a change in beta and/or gamma oscillations would also be observed as the sentence unfolded.

\section{Methods}

\section{Participants}

A total of 102 native Dutch speakers (51 males), with an age range of 18 to 33 years (mean of 22 years), participated in the experiment. These participants formed part of a larger study-MOUS (Mother of all Unification Studies; $N=204$ ), where all participants took part in an fMRI and a MEG session. Half of these participants completed both sessions where they read the stimuli, and the other half listened to recordings of the 
stimuli. The current paper pertains to participants from the MEG session in the visual modality. All participants were right-handed, had normal or corrected-to-normal vision, and reported no history of neurological, developmental, or language deficits. The study was approved by the local ethics committee (CMO-the local "Committee on Research Involving Human Participants" in the Arnhem-Nijmegen region) and followed the guidelines of the Helsinki declaration. Participants received monetary compensation for the participation.

\section{Language stimuli}

The stimuli consisted of 180 sentences and their word list counterparts (see Table 1 for an example). All sentences varied between 9 and 15 words in length.

The word lists were created from the sentences by scrambling the words so that no more than two consecutive words formed a coherent fragment. The same words in both conditions limited the difference between conditions to sentential semantics and syntax as opposed to lexical differences in orthography, phonology, and word meaning. Each participant saw each stimulus in either the sentence or the word list condition, but not in both. Across participants, each stimulus was presented the same number of times in the sentence and in the word list condition.

\section{Task and procedure}

\section{Experimental design}

All stimuli were presented with an LCD projector (with a vertical refresh rate of $60 \mathrm{~Hz}$ ) situated outside the MEG measurement room, and projected via mirrors onto the screen inside the MEG room. All stimuli were presented at the center of the screen within a visual angle of 4 degrees, in a black mono-spaced font, on a gray background using Presentation software (Version 16.0, Neurobehavioral Systems, Inc).

The stimuli were divided into three subsets, such that each participant saw $2 / 3$ of the stimulus set in the MEG session (120 trials of each condition); and $1 / 3$ in the fMRI session that will not be further discussed in this paper. Participants presented with the same subset saw the stimuli in a different (randomized) order. In the experiment, the stimuli were presented in a mini block design, and alternated between a sentence block (containing 5 sentences) and a word list block (containing 5 word lists), for a total of 24 blocks. The first mini block (sentences or word lists) was randomized across participants. In addition, for sentences, the first word began with a capital letter and the last word ended with a full stop.

At the beginning of each block, the block type was announced for 1500 ms: zinnen (sentences) or woorden (words), followed by a 2000 ms blank screen. At the beginning of each trial, a fixation cross was presented for a jittered duration between 1200 and 2200 ms. Then, the words for each trial (sentence or word list) were presented one at a time. Each word was separated by a blank screen for $300 \mathrm{~ms}$.

The presentation time of each word was varied in order to allow for a 'naturalistic' reading experience, and to avoid a strict entrainment of ongoing activity to fixed interstimulus intervals. For any given sentence (or word list) the variable duration of a single word was a function of the following quantities: (i) the total duration of the audio-version

Table 1

Exemplar sentence and word list in Dutch, and literal English translation.

\begin{tabular}{ll}
\hline Sentence & Word list \\
\hline $\begin{array}{l}\text { Bij de opening van de nieuwe sporthal } \\
\text { kregen de talrijke bezoekers een }\end{array}$ & $\begin{array}{l}\text { sporthal bij van talrijke opening een de de } \\
\text { kregen consumptie bezoekers nieuwe de }\end{array}$ \\
$\begin{array}{ll}\text { At the opening of the new sports hall } \\
\text { received the many visitors a (free) } \\
\text { drink }\end{array}$ & $\begin{array}{l}\text { sports hall at from many opening a the the } \\
\text { received (free) drink visitors new the }\end{array}$ \\
\hline
\end{tabular}

of the sentence/word list (audiodur), (ii) the number of words in the sentence (nwords), (iii) the number of letters per word (nletters), and (iv) the total number of letters in the sentence (sumnletters). Specifically, the duration (in ms) of a single word was defined as: (nletters/ sumnletters)*(audiodur $+2000-150^{*}$ nwords). The minimum duration of short words was set to $300 \mathrm{~ms}$ irrespective of the relative weighting described by the formula. In practice, however, the exact presentation times of the words slightly deviated from those obtained from the above formula. This was due to the fact that the presentation timing was dictated by the refresh rate of the projector $(60 \mathrm{~Hz})$. As a consequence, the actual presentation time was lengthened by a duration between 0 and $33 \mathrm{~ms}$. The median duration of a single word on the screen was $434 \mathrm{~ms}$ (range 300-1344 ms). Taking into account the $300 \mathrm{~ms}$ gap between the words, the median duration of a whole sentence/word list was $8.3 \mathrm{~s}$ (range 6.2-12 s). Within each block, the inter-trial interval was a blank screen with a jittered duration between 1200 and 2200 ms.

In order to check for compliance, $10 \%$ of the trials $(n=12)$ were randomly followed by a yes/no question about the content of the previous sentence/word list. Half of the questions on the sentences addressed sentence comprehension (e.g., Did grandma eat a pancake?). The other half of the sentences, and the questions following the word lists addressed a content word (e.g., Was a music instrument mentioned?). Participants answered the question by pressing a button for 'Yes'/“No' with their left index and middle fingers, respectively. For both question types, half of the trials had a yes-response as the correct answer. The experiment began with participants reading written instructions for the task. The experimenter clarified any questions from the participant. Then, for familiarization purposes, participants completed a practice task (using a separate set of stimuli from the actual task). Subsequently, they performed the actual task as described above.

\section{MEG data acquisition}

MEG data were collected with a 275 axial gradiometer system (CTF) at the Donders Centre for Cognitive Neuroimaging in Nijmegen, The Netherlands. The signals were digitized at a sampling frequency of $1200 \mathrm{~Hz}$ (the cutoff frequency of the analog anti-aliasing low pass filter was $300 \mathrm{~Hz}$ ). Three coils were attached to the participant's head (nasion, left and right ear canals) to determine the position of the head relative to the MEG sensors. Throughout the measurement, the head position was continuously monitored using custom software (Stolk et al., 2013). During breaks, the participant was allowed to reposition to the original position if needed. Participants were able to maintain a head position within $5 \mathrm{~mm}$ of their original position. Three bipolar $\mathrm{Ag} / \mathrm{AgCl}$ electrode pairs were used to measure the horizontal and vertical electro-oculogram, and the electro-cardiogram.

\section{Data processing}

All analyses were done with custom written MATLAB scripts and FieldTrip, an open source toolbox for EEG and MEG data analyses (Oostenveld et al., 2011).

\section{Preprocessing}

Electrocardiogram artifacts were estimated using denoising source separation (DSS, Särelä and Valpola, 2005), identified based on their topography and subtracted from the data. Physiological artifacts (eye movements and muscle contractions) and jump artifacts in the SQUIDs (superconducting quantum interference device) were identified using a semi-automatic artifact identification procedure (http://www. fieldtriptoolbox.org/tutorial/automatic_artifact_rejection), followed by visual inspection. Data segments that contained artifacts were excluded from further analysis. Next, the power line interference at $50 \mathrm{~Hz}$ and its harmonics at 100 and $150 \mathrm{~Hz}$ were estimated and subtracted from the data (for details see Schoffelen et al., 2005). Finally, the data were downsampled to a sampling frequency of $300 \mathrm{~Hz}$. 


\section{Frequency-domain beamforming}

We used a frequency domain beamformer (DICS; Gross et al., 2001) to reconstruct the sources of the oscillatory responses. First, for each time window and frequency band, the sensor-level cross-spectral density matrix was computed across all conditions. The frequency bands were selected based on earlier studies. For the low frequencies, they were centered at the following frequencies (with effective spectral bandwidth in brackets): $5 \mathrm{~Hz}(3.75-6.25 \mathrm{~Hz}$ ) for theta, $10 \mathrm{~Hz}$ $(8.75-11.25 \mathrm{~Hz})$ for alpha, and $16 \mathrm{~Hz}(14-18 \mathrm{~Hz})$ for beta. Here, each discrete frequency designates the centre frequency of a band-limited frequency bin, and this centre frequency encompasses the average signal power across the frequency range in that bin, referred to as the effective bandwidth. This bandwidth is dictated by the combination of the chosen window length and tapering scheme. The theta and alpha band were analyzed in $50 \mathrm{~ms}$ time steps from -100 to $500 \mathrm{~ms}$ (around word onset at $0 \mathrm{~ms}$ ) using a sliding time window of $400 \mathrm{~ms}$ in combination with a Hanning taper, which produced an effective bandwidth of $2.5 \mathrm{~Hz}$ (which is $1.25 \mathrm{~Hz}$ around the defined centre frequencies). Beta was also analyzed in $50 \mathrm{~ms}$ time steps from -150 to $500 \mathrm{~ms}$, but with a $250 \mathrm{~ms}$ time window in combination with a Hanning taper, which produced an effective bandwidth of $4 \mathrm{~Hz}$. The higher frequencies, were a priori defined as $36-76 \mathrm{~Hz}$ (low gamma), and 76-108 Hz (high gamma), and thus encompassed spectral estimates across multiple frequency bins. We analyzed them at $50 \mathrm{~ms}$ time steps, at $4 \mathrm{~Hz}$ steps, between -150 and $500 \mathrm{~ms}$. Here, we used a $250 \mathrm{~ms}$ time window in combination with multitapers (Mitra and Pesaran, 1999), which achieved an effective bandwidth of $16 \mathrm{~Hz}$.

The sensor-level cross-spectral density matrix was then used in combination with the forward solution to compute a set of spatial filters to obtain an estimate of the activity for dipoles placed on a volumetric grid with $\sim 8 \mathrm{~mm}$ spacing (see below). The spatial filters assumed a fixed orientation of the underlying dipoles, defined by means of a singular value decomposition of the dipole cross-spectral density, taking the orientation along the first singular vector to explain the maximum amount of variance. The activity (power) was estimated for each condition of interest.

Processing of the anatomical MRI and digitized headshape for MEG source reconstruction

For source reconstruction purposes, we coregistered the anatomical MRI to the MEG sensors. This was achieved by manual alignment of two reconstructions of the head surface. A digitized head shape was obtained with a Polhemus device (a 3D digitizer from Fastrak, Polhemus Inc., Colchester, VA, USA). This consisted of approximately 500 points across the scalp and was used to reference the location of MEG sensors relative to the head (and the fiducials). The second head shape was created from the participant's T1-weighted anatomical MRI image. Subsequently, the aligned anatomical image was used to create (i) a volume conduction model based on a single shell description (Nolte, 2003) of the inner surface of the skull, using the segmentation function in SPM8, (ii) a set of spatial normalization parameters to bring each participant's brain into a normalized volumetric space, using SPM8, and (iii) a description of the cortical surface, using Freesurfer 5.1 (Dale et al., 1999). The spatial normalization parameters were used to create individual volumetric grids which in turn were used for a beamformer source reconstruction of the oscillatory responses. A template volumetric grid with a resolution of $8 \mathrm{~mm}$ was warped into individual brain space, using the inverse of the normalization parameters.

\section{Statistical analysis}

The frequency bands selected for source analysis were also used for statistical inference. For each frequency band, statistical inference was done for a selected set of time windows (indicated below in each analysis) using a non-parametric permutation test together with a clustering method (Maris and Oostenveld, 2007), to correct for multiple comparisons.

For the observed data, a dependent samples $t$-statistic for the difference between the conditions was computed for each sample (spacefrequency-time point). For each of the lower frequencies, this yielded a single 3-dimensional volumetric map of $t$-values for each of the time windows tested. For the gamma range, this yielded 4-dimensional volumetric maps of $t$-values (three spatial dimensions + frequency). Samples that exceeded the uncorrected significance level of $1 \%$ were clustered according to adjacency (in the spatial domain for theta, alpha, and beta, and in the spatial and frequency domain for the gamma band). For each cluster, the cluster-level $t$-statistic was calculated by summing the $t$-statistics across the individual elements. Next, to test the observed $t$-statistic, a reference distribution was computed. This distribution was created by permuting (randomly exchanging) data between the conditions, and then calculating the maximal positive and negative cluster-level $t$-statistic for each permuted data set. Finally, the observed $t$-statistic was tested against the reference distribution. The observed $t$-statistic was considered significant if it was located beyond the determined threshold, on the negative or positive end of the reference distribution. The statistical threshold is specified in the section of each analysis below (see Word analysis and Context analysis).

To address the multiple tests performed across 3 time windows and 5 frequency bands, we applied a conservative Bonferroni correction to the critical threshold to infer statistical significance from the $p$-values. The details on this correction are provided below in each analysis section. For visualization, the thresholded volumetric images were interpolated onto the cortical sheet extracted from the MNI template brain, exported as a cifti-file and displayed using the workbench software package (Marcus et al., 2011).

\section{Word analysis}

To compare the condition-specific (sentences or word lists) response to individual words, for each condition, we first subtracted the estimated power in the period preceding word onset (baseline) from the power following word onset. Changes in oscillatory activity in response to a single word require time to develop, and probably more than $100 \mathrm{~ms}$ to become apparent. Therefore, the choice of a pre-word baseline allowed us to study word-induced changes in frequencyspecific activity, above and beyond the ongoing nature of oscillations. Furthermore, it mitigated the possible effect of a block design producing different pre-window activity for each condition. For the alpha and theta bands, we used a baseline estimate centered at $-100 \mathrm{~ms}$. For the beta and gamma bands, the baseline estimate was an average of two time windows centered at -150 and $-100 \mathrm{~ms}$. As a consequence, the estimated power at these time windows included a short duration of data following word onset (up to $100 \mathrm{~ms}$ for the theta band, and up to $25 \mathrm{~ms}$ for all other frequencies). We did not use an earlier time window, e.g., $-200 \mathrm{~ms}$, because this would potentially include transient oscillatory activity from the previous word. To study the neural response to each word, we chose three time windows following word onset: centered at 250, 350, and $450 \mathrm{~ms}$, referred to as the first, second, and third time windows, respectively. We excluded the initial $250 \mathrm{~ms}$ because previous studies have suggested that this is the time window of visual processes (Dehaene et al., 2002; Tarkiainen et al., 1999) while the majority of higher order processes, which are the main focus of our interest, such as lexical and context effects, occur later in time (e.g., Vartiainen et al., 2009). As visual inspection of the oscillatory power time courses (Fig. 1) revealed variations in differences between conditions across time windows, we chose to statistically test the data for each time separately.

To address how words are processed in a sentence, we compared words embedded in a sentence to words embedded in a word list. For each participant, we analyzed the averaged single-word response (power) for each condition. The number of epochs available for averaging was different across conditions, due to artifact rejection. To avoid 


\section{Word Analysis}

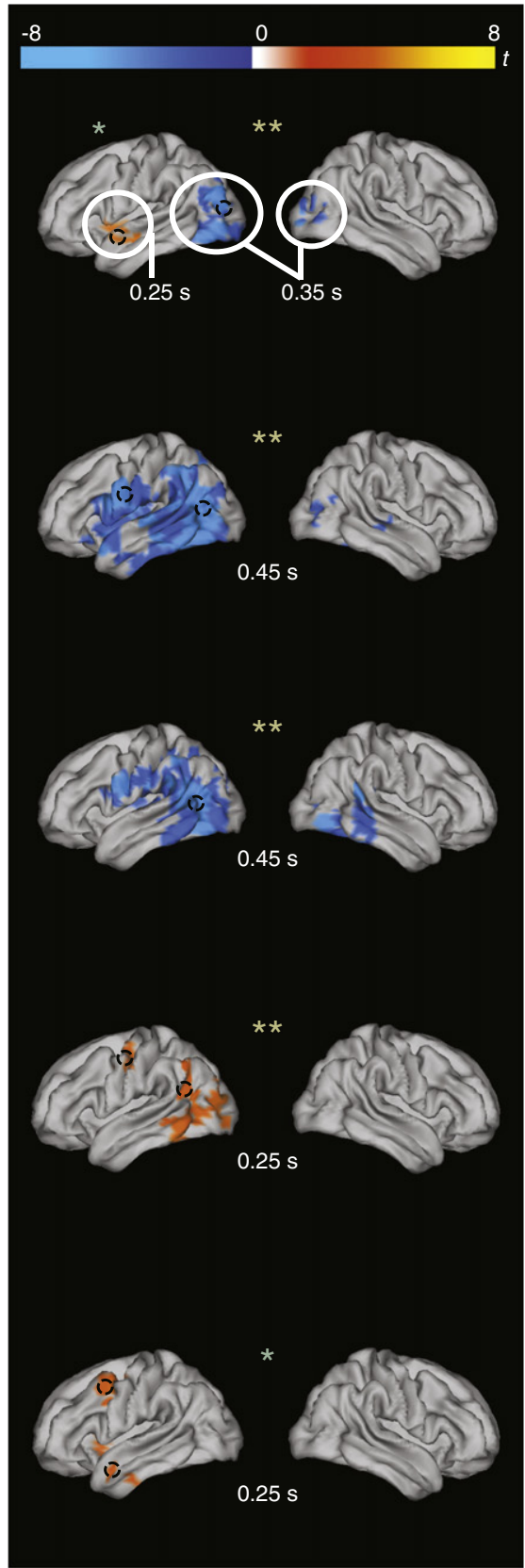

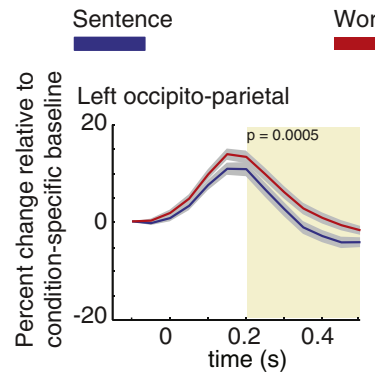

Alpha

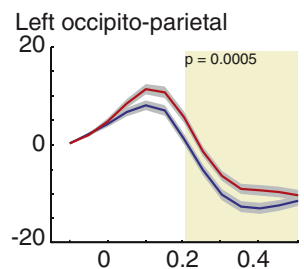

Beta

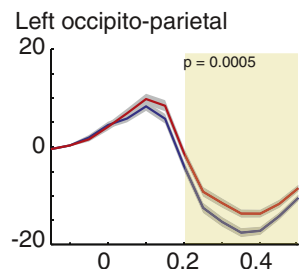

Low

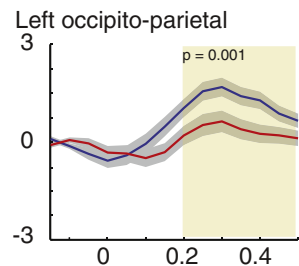

High

Gamma

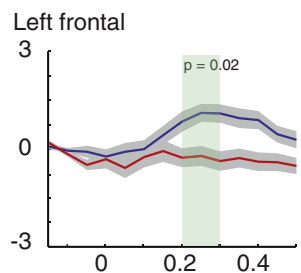

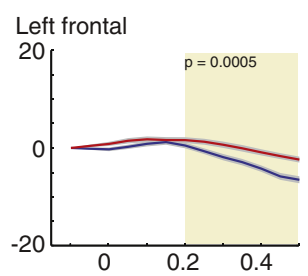
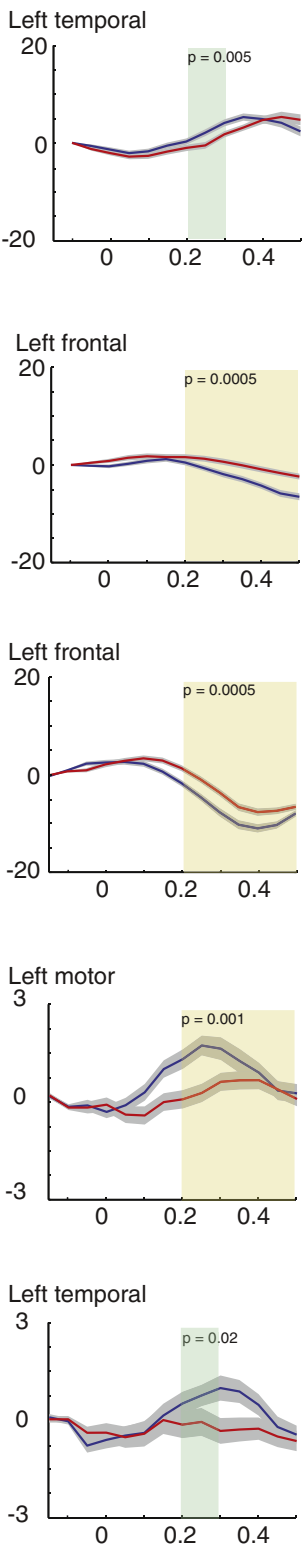

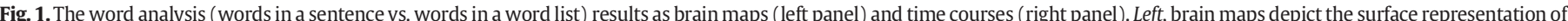

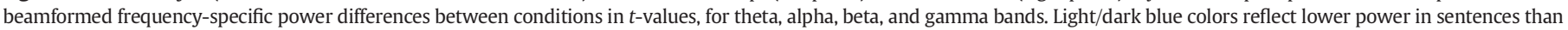

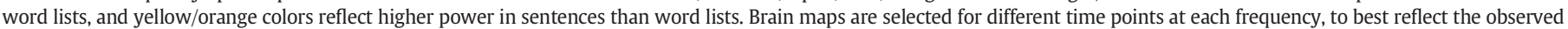

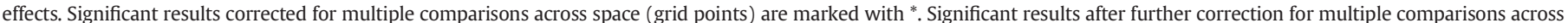

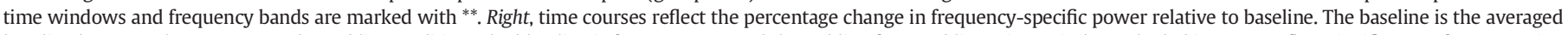

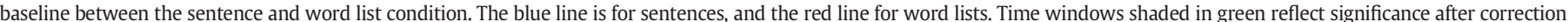

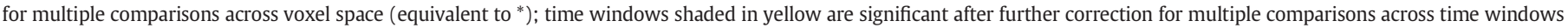

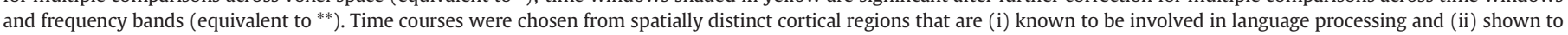

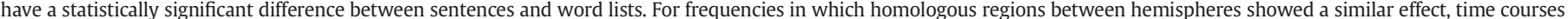

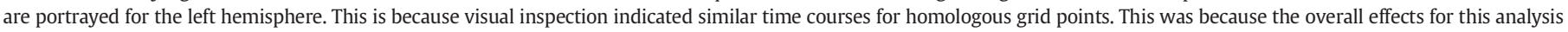
were left hemisphere dominant. Black circles on the brain map indicate the region of the chosen grid points.

effects due to differences in signal to noise ratio, we equalized the number of epochs across conditions for each participant. For each of the 3 time windows and 5 frequency bands, we performed a separate test. In our tests we applied a conservative Bonferroni correction, and used a corrected critical $p$-value of $0.0017(0.025 / 15$, for a twosided test).

\section{Context analysis}

In this analysis, we were interested in the change in neural activity as the sentence unfolded. To do this, we quantified the difference in the neural response to late versus early words in a sentence and in a word list. We accounted for non-specific variability in overall power values across participants by subtracting a condition-averaged estimate 
of the power preceding word onset from each of the condition-specific power following word onset, for each participant. Note that the subtraction of the same 'baseline' will yield the same average difference across participants, in comparison to contrasting the raw power values. As in the word analysis, for the context analysis, we chose the same time windows following word onset (centered at 250,350, and $450 \mathrm{~ms}$ ) for analysis.

The statistical analysis here addressed the effect of an incremental context on word processing. We assessed the neural activity during word processing as a function of word position across a sentence. We extracted the early words (2nd, 3rd, and 4th word positions) and the late words (n-2nd, $n$-3rd, and $n$-4th word positions; where $n$ is the total number of words in a sentence/word list) in both sentences and word lists, resulting in four conditions (sentence-early, SE; sentencelate, SL; word list-early, WE; and word list-late, WL). To control for lexical frequency, we used a stratification approach. First, the lexical frequency value for each word was determined, using the SUBTLEX-NL database of Dutch word frequencies (Keuleers et al., 2010), and log transformed. Then, for each condition, a histogram of log-transformed lexical frequencies was created, using 10 bins. For each of the bins, we determined the minimum number of contributing trials across conditions. Subsequently, trials were randomly removed from each bin of each condition to meet the minimum, which produced the same number of trials per bin per condition. This yielded about 200 trials per condition. Subsequently, we calculated an average power for each condition.

To test for significance, we first determined whether there was an interaction between word position (late vs. early) and sentence type (sentence vs. word lists): ([SL-SE] - [WL-WE]), at each of the five frequency bands. To correct for doing multiple tests, we applied a Bonferroni-corrected threshold of $p<0.005$. For frequencies showing a significant interaction, we performed a second statistical test for each condition: SL-SE and WL-WE, to determine an effect of late vs. early context within each condition. Here, we visually inspected the power time courses, and found a consistent power difference between conditions over time (Fig. 2). Therefore, we chose to perform one test for each frequency, across all time windows. In addition, to determine whether the late vs. early context effect was specific to sentences, we performed post hoc tests comparing SL-WL and SE-WE. Here, we selected an individual grid point for each region that showed the most prominent, significant difference for the SL-SE contrast. We selected grid points from (i) spatially distinct regions, and (ii) regions involved in language processing. In the case where similar regions were activated in both hemispheres, as indicated by visual inspection of homologous grid points, we chose the grid point from the hemisphere that showed a stronger effect. All grid points are indicated with black circles in Fig. 2.

\section{Results}

\section{Task compliance}

The mean percentage of correct answers for questions that followed a sentence or word list was $81.1 \%(S D=6.7 \%$ ). Performance was significantly higher for sentence questions on a main content word
$(M=84.2 \%, \mathrm{SD}=11.6 \%)$ than for a word list question $(M=78.4 \%$, $\left.\mathrm{SD}=9.6 \% ; t_{101}=5.75, p<0.001\right)$. Potentially, the difference in performance was because sentences are easier to process than word lists. Nevertheless, with all participants performing well above chance in both cases, they were attentive and processed the language materials. Note that there was no difference between the sentence comprehension questions $(M=83 \%, \mathrm{SD}=11.7 \%)$ and the sentence content word questions $\left(t_{101}=-1.2, p=0.4\right)$.

\section{Word analysis}

In the word analysis, we compared whether individual words in a meaningful and structured context were processed differently from words in a word list. We observed significant differences between sentences $(\mathrm{S})$ and word lists $(\mathrm{W})$ in the theta, alpha, beta, and gamma frequency bands in multiple brain regions, as shown in Fig. 1. The oscillatory power time courses (as a relative percent change to a baseline averaged over conditions; see Fig. 1) suggested that for most frequencies, the effect was driven by a difference in magnitude and not shape of the time course, with the exception of left temporal theta. In general, differences between conditions resulted in an ERD for frequencies below $40 \mathrm{~Hz}$ (except for left temporal theta), and an ERS for frequencies above $40 \mathrm{~Hz}$. Here, we focus on the statistically significant results, and will refer to the first ( $250 \mathrm{~ms})$, second $(350 \mathrm{~ms})$, and third $(450 \mathrm{~ms})$ time windows, relative to word onset. For an in-depth description and visual depiction of the spatiotemporal evolution of frequency-specific power, the interested reader is directed to supplementary material 1.

\section{Theta band}

Significant differences were found in the bilateral occipital cortex, and left posterior temporal regions in all three time windows $(\mathrm{S}<\mathrm{W}$; $p=0.0005$, corrected for multiple comparisons). The left temporal region in the first time window was also significant but only when uncorrected for multiple comparisons, $(p=0.005, S>W$; Fig. 1$)$.

\section{Alpha band}

A significant difference was found in all three time windows, in the bilateral occipito-parietal, left frontal, and left temporal regions ( $p=0.0005, \mathrm{~S}<\mathrm{W}$; corrected for multiple comparisons).

\section{Beta band}

A significant difference was found in all three time windows, in the left occipito-parietal cortex, left posterior temporal areas, and left frontal cortex ( $p=0.0005, \mathrm{~S}<\mathrm{W}$; corrected for multiple comparisons).

\section{Gamma band}

In the gamma band, effects were more left lateralized than in the lower frequency bands. Across time windows, we observed a posterior to anterior activation sweep (Fig. 1). Visual inspection revealed that low and high gamma showed distinct spatiotemporal dynamics. The statistical test for low gamma $(40-68 \mathrm{~Hz})$ revealed a significant difference only in the first time window in left occipital, left parietal, left motor, and left temporal regions $(p=0.001, \mathrm{~S}>\mathrm{W}$; corrected for multiple comparisons). In the subsequent time windows, the effect

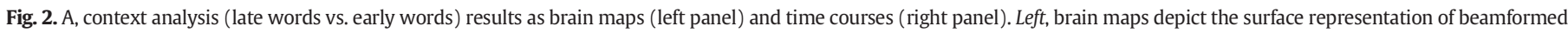

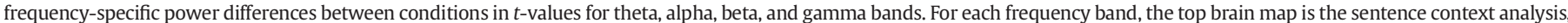

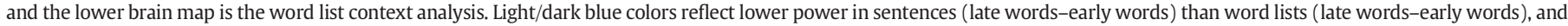

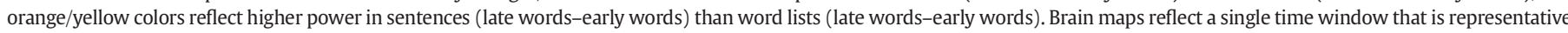

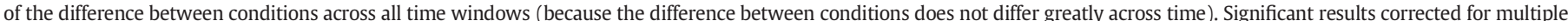

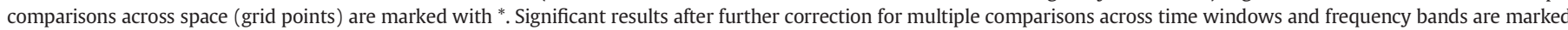

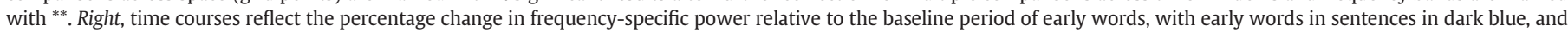

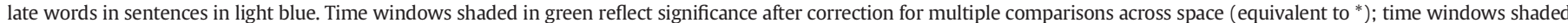

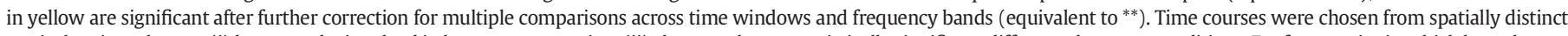

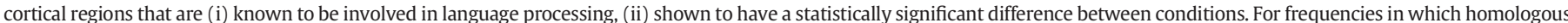

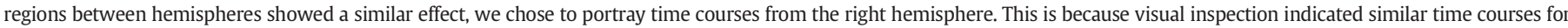

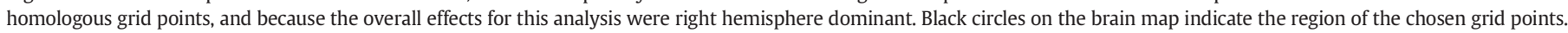
$\mathrm{B}$, Bar graphs depicting sentence early words vs. word list early words, and sentence late words vs. word list late words. Error bars reflect \pm 1 standard error of the mean.
} 


\section{Context Analysis}
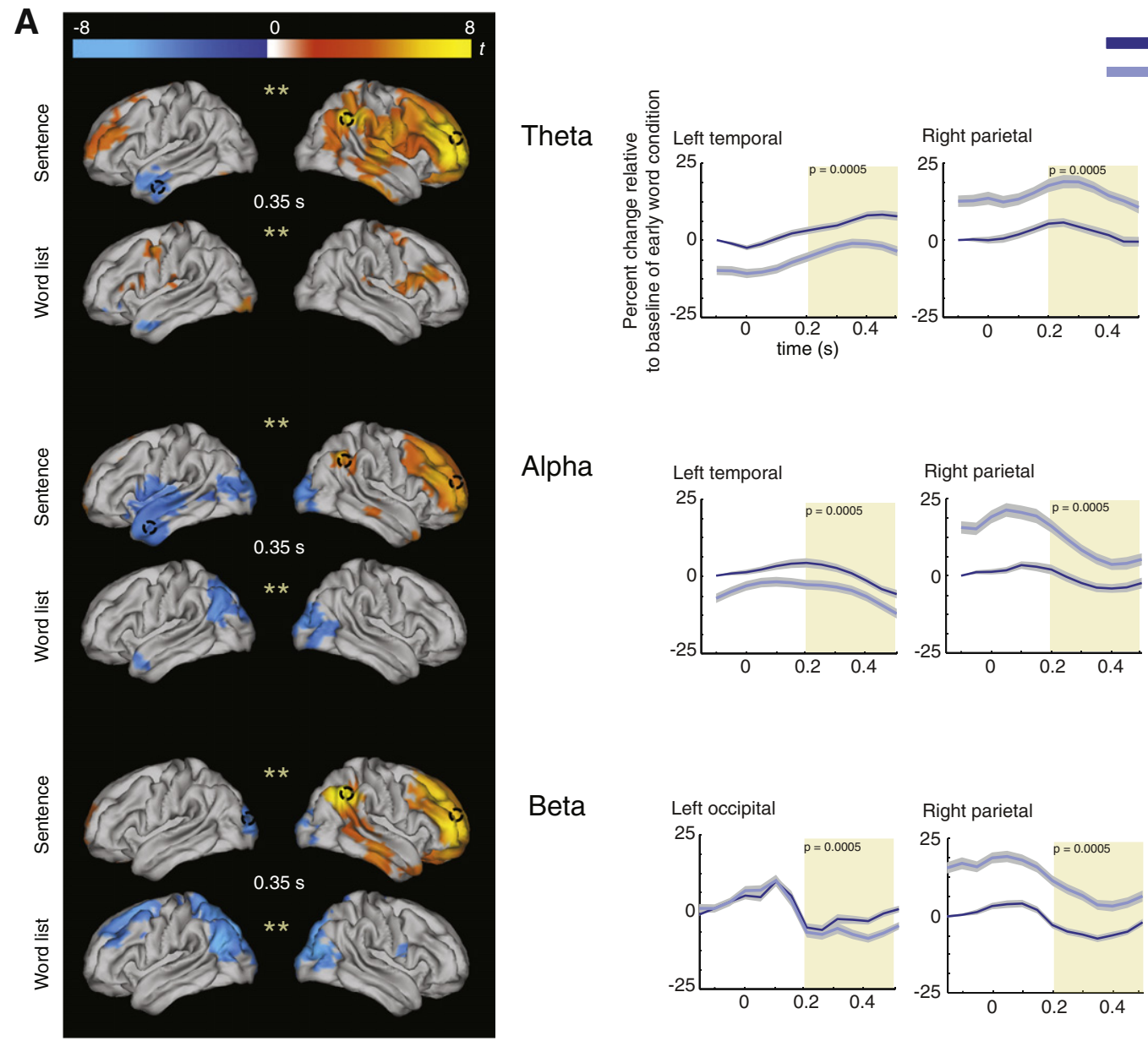

Early words in sentence

Late words in sentence

Alpha
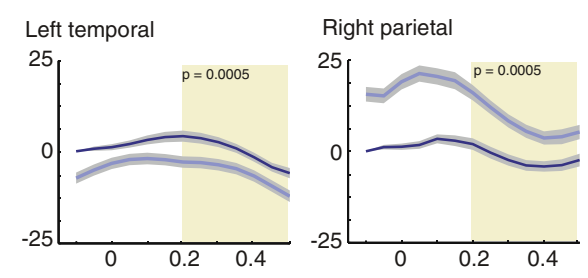

Right frontal
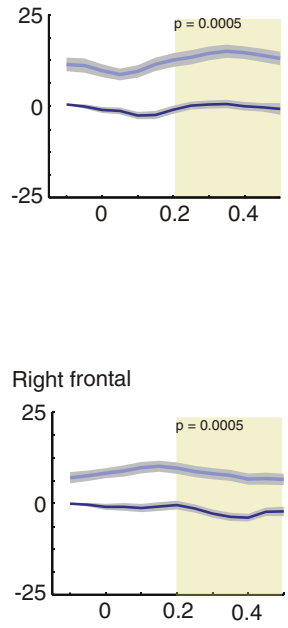

Beta
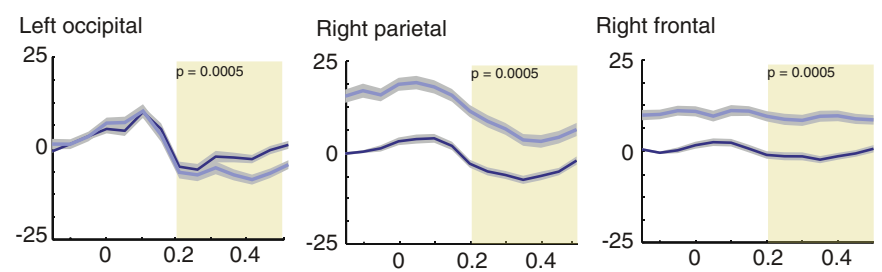

B Theta Left temporal

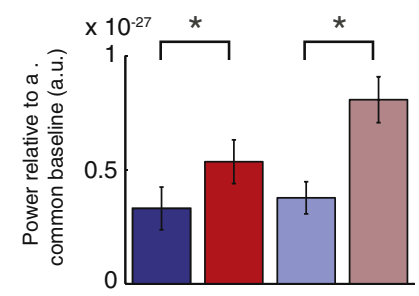

Alpha Left temporal

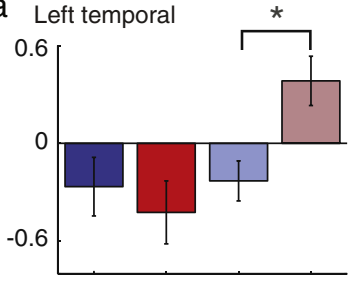

Beta

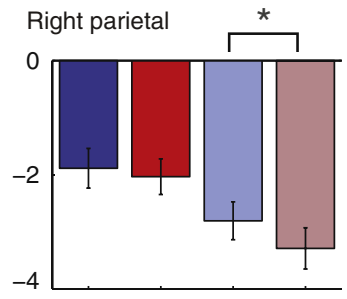

Right parietal
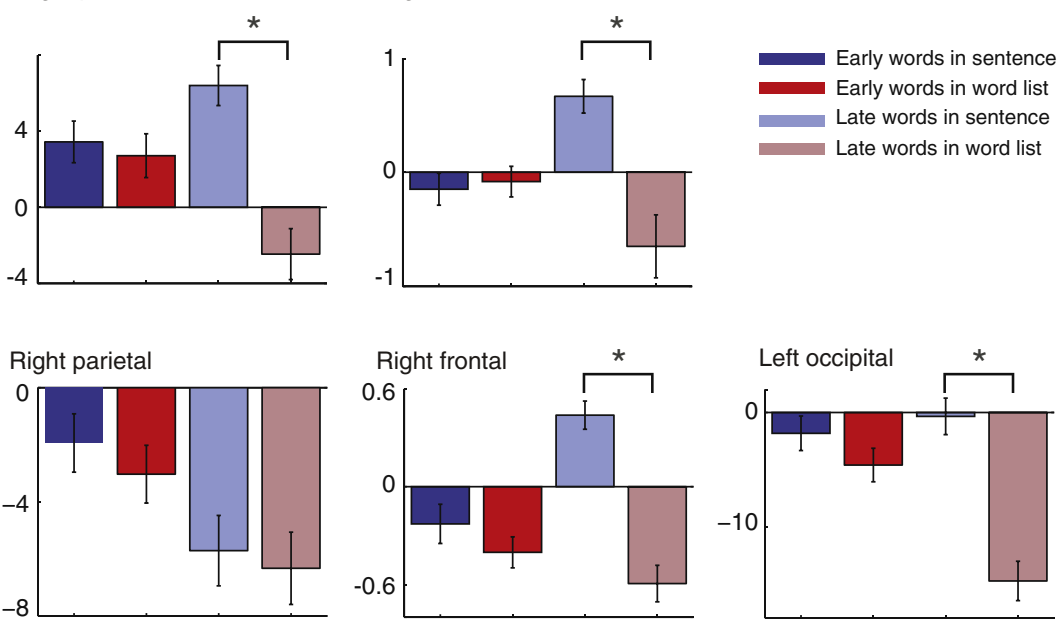

Right frontal

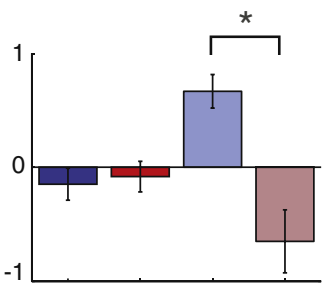

Early words in sentence Early words in word list Late words in sentence Late words in word list

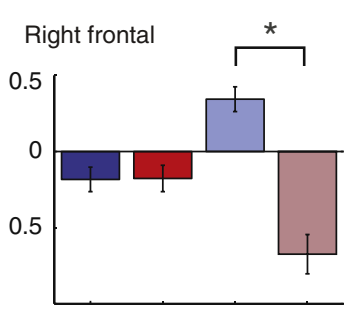


reduce in effect size $(p=0.008, \mathrm{~S}>\mathrm{W})$. The statistical test for high gamma $(84-100 \mathrm{~Hz})$ revealed a significant difference in left frontal and temporal regions when uncorrected for multiple comparisons $(p=0.02, \mathrm{~S}>\mathrm{W})$.

\section{Context analysis}

The context analysis focused on how oscillatory activity changes as the sentence unfolds. The accumulation of words in a sentence, but not a word list, should form a more meaningful and structured context to facilitate the processing of each subsequent word. Here, we assessed whether there was a change in oscillatory power between late and early words in a sentence, and compared this to the same contrast in word lists. Early words were the 2nd, 3rd, and 4th words in a sentence (or word list), and late words were the 4th, 3rd, and 2nd to last words in a sentence (or word list). The interaction analysis [S(L-E) - W(L-E)] for theta, alpha, and beta was significant $(p<0.0083)$. Therefore, for each condition, we compared late versus early words, within each condition (SL-SE, and WL-WE) across all three time windows. In all three frequency bands, late words were significantly different from early words (context effect) for sentences, and word lists, but the effects were stronger for sentences than word list (Fig. 2), details for each frequency are reported in the following sections.

As a complementary test to the one above, we contrasted late words in sentences with those in word lists (SL-WL; late word comparison), and contrasted early words in sentences with those in words lists (SE-WE; early word comparison). This demonstrated that the difference between late and early words was predominant in sentences, details reported below for each frequency (Fig. 2). A detailed description and depiction of the evolution of power over time is available in the supplementary materials (Fig. S1b). Two alternative analyses for the context effects are presented in the supplementary materials (see sentence progression analysis and adapted context analysis; Fig. S2).

\section{Theta band}

For sentences, we observed statistically significant context effects in bilateral frontal and right parietal regions $(\mathrm{L}>\mathrm{E})$ as well as in left anterior temporal regions ( $\mathrm{L}<\mathrm{E} ; p=0.005$; corrected for multiple comparisons). In word lists, the context effect was significant in areas similar to the context effect in sentences ( $p=0.005$; corrected for multiple comparisons). However, the spatial extent was limited, the difference in power was smaller, and the difference remained constant across time. The test for SL-WL and SE-WE were only significant $(p<0.025)$ for the late word comparison in grid points in right parietal and right frontal regions. However, in the left anterior temporal region, these effects were significant $(p<0.025)$ in both the early word and late word comparisons.

\section{Alpha band}

For sentences, we observed a significant difference in left temporal and inferior frontal regions $(\mathrm{L}<\mathrm{E} ; p=0.005$; corrected for multiple comparisons) as well as in bilateral frontal, right parietal, and right temporal regions ( $\mathrm{L}>\mathrm{E} ; p=0.005$; corrected for multiple comparisons). For word lists, significant differences were observed in the left temporal and bilateral occipito-parietal regions ( $\mathrm{L}<\mathrm{E} ; p=0.005$; corrected for multiple comparisons) as well as right temporal and right frontal areas ( $\mathrm{L}>\mathrm{E}$; $p=0.005$; corrected for multiple comparisons). These differences were spatially more focal than those in the sentence condition. The test for SL-WL and SE-WE were only significant $(p<0.025)$ for the late word comparison in grid points in left occipital, left temporal, right parietal, and right frontal regions.

\section{Beta band}

For sentences, we observed a significant difference in bilateral frontal, right parietal, and right temporal regions ( $\mathrm{L}>\mathrm{E} ; \mathrm{p}=0.005$; corrected for multiple comparisons) as well as in bilateral occipital regions ( $\mathrm{L}<\mathrm{E} ; p=0.005$; corrected for multiple comparisons). For word lists, bilateral occipital, parietal, and middle frontal regions were significant for all time windows $(p=0.005$; corrected for multiple comparisons). The test for SL-WL and SE-WE were only significant $(p<0.025)$ for the late words comparison in grid points in left occipital, right parietal, and right frontal regions.

\section{Discussion}

In this study, we investigated neuronal oscillatory activity during sentence processing with MEG. We used source reconstruction techniques to quantify the spatiotemporal response in five well-established frequency bands. The word analysis determined how individual words are processed in a sentence by comparing the oscillatory response to words embedded in a sentence (i.e. within a syntactically and semantically meaningful context) to that of a word list (which lacked a structured context). To determine the effect of an incremental context on sentence processing, the context analysis quantified the changes in the word-induced modulations of oscillatory activity at different stages of the unfolding sentence. Here, we focused on the context effect (late versus early words) in sentences, and compared it to the context effect in word lists as a control. We evaluated which regions and time-frequency points showed significantly more or less activity in the sentence than word list context analysis.

The word analysis revealed the effects of (a sentence) context on word processing in the theta, alpha, beta, and gamma bands, predominantly in the left hemisphere inferior frontal, temporal, and parietal regions. The context analysis captured effects of the incremental context in left temporal and occipito-temporal regions, and in right-lateralized frontal and parietal regions in the theta, alpha, and beta bands. We consider the findings from these two comparisons to be complimentary and will discuss them in light of each other.

Task-related modulations of oscillatory activity can occur in multiple frequency bands that vary in the direction of the effect (ERS or ERD). This heterogeneity across frequency bands poses challenges to the interpretation of the findings. Common practice in cognitive studies has been the attribution of a specific functional role to a specific frequency band within the given experimental context. However, adopting such strict task-related functional interpretations of particular frequencies might be too ambitious given that different types of rhythmic activity occur in multiple brain regions, across multiple temporal and spatial scales. Moreover, these rhythms may not be specific to any task. Here, we propose a set of explanations that account for the various effects by taking into account the neural areas and frequencies of the observed effects, and previous studies that analyzed oscillations in the context of language and non-language tasks.

\section{Theta oscillations in sentence processing}

In the theta band, we observed effects in the word and context analyses in left anterior temporal regions.

Stronger theta synchronization was present for sentences compared to word lists in the word analysis. Inspection of the time course of theta activity suggests that this difference reflects a latency shift, with theta peaking earlier in sentences (around $350 \mathrm{~ms}$; word lists around $400 \mathrm{~ms}$ ). We tested this latency shift post hoc. This latency shift was significant across subjects (Wilcoxon signed-rank test, $Z=-2.84$ $p=0.004)$. The observation of this theta synchronization soon after word onset (around $200 \mathrm{~ms}$ ) for both sentences and word lists could indicate a common, early process, most likely word retrieval. Since the theta peak is earlier for sentences than word lists, this suggests a stronger facilitatory context effect on lexical retrieval for sentences compared to word lists.

In the context analysis, for sentences and word lists, late words compared to early words were associated with a decrease in theta power in left anterior temporal regions. We speculate that this lower theta 
activation is the effect of an incrementally more informative context which increases the facilitatory effect on retrieval as the sentence (or word list) unfolds. As each word list was created by scrambling a sentence, the words in a word list also had an associative relation at the lexical level, which could provide an associative context for upcoming words, and therefore a context facilitatory effect on retrieval. Importantly, because sentences are grammatical and meaningful, they have stronger constraints and thus a higher predictive value for the upcoming word, which results in a stronger context facilitatory effect for sentences than word lists. In line with our interpretation, many previous studies have shown that left anterior temporal regions are associated with conceptual relations between words at the lexical level (e.g., Mummery et al., 2000; Patterson et al., 2007). Together, these findings suggest that having a context allows for easier lexical retrieval, and when this context becomes more informative (more preceding words in a sentence context) less cognitive resources are needed for lexical retrieval.

Our interpretation of theta band effects in left temporal regions as being associated with lexical retrieval is in line with other oscillatory studies on memory. Memory retrieval (or attempted retrieval) has resulted in theta band synchronization in response to language stimuli (single words, word in a sentence) and non-language stimuli (e.g., shapes and faces) with a widespread sensor-level topography (Bastiaansen et al., 2002; Klimesch et al., 2008, 2010; Mormann et al., 2005; Osipova et al., 2006). At the source-level, an earlier study on memory retrieval localized effects to medial temporal, prefrontal, and visual areas (Guderian and Düzel, 2005), which is a partial overlap with our findings. The additional areas reported by this earlier study could be due to the type of information being retrieved, since their study used faces and a background scene, whereas we used words.

In bilateral frontal and right parietal regions, we also observed theta effects specific to the context analysis: theta power was significantly higher for late words than early words in sentences. The spatial topography of our results is similar to the frontal-parietal network associated with cognitive task demands (Chein et al., 2011; Fedorenko et al., 2013). Words late in the sentence are likely to increase task demand, since a more extended context representation has to be maintained in memory. In line with previous studies, an increase in theta synchronization in frontal and parietal sensors has been observed with an increase in working memory load (e.g., Cashdollar et al., 2009; Deiber et al., 2007; Jensen and Tesche, 2002). In addition, active maintenance of item(s) increases theta synchrony between right frontal and parietal regions (Cashdollar et al., 2009; Deiber et al., 2007). Support also comes from a language study that found an increase in theta power in rightcentro parietal MEG sensors as a sentence unfolded (Bastiaansen et al., 2010), which was thought to reflect the memory trace of the unfolding sentence representation.

In bilateral occipito-temporal regions, we observed a theta effect in the word analysis. Theta power was significantly lower in bilateral occipito-temporal regions for words in a sentence versus words in a word list. The difference begins around $100 \mathrm{~ms}$, becomes larger and remains stable over time. Potentially, this reflects a difference in visual word recognition between sentences and word lists. Specifically, the sentence context narrows down certain aspects of the upcoming word (e.g., word category, semantics) which could modulate the response in occipital regions to the incoming word. The neural mechanism underlying this modulation may be top-down influences from higher to lower cortical regions. Studies on visual and motor processing have shown that neural activity in response to a visual stimulus in occipital cortex can be directly modulated by activity from frontal and parietal regions (Engel et al., 2001; Silvanto et al., 2009; Taylor et al., 2007). In one case, transcranial magnetic stimulation of the posterior parietal cortex led to an increase in primary visual cortical excitability (Silvanto et al., 2009). We speculate that the larger desynchronization for words in a sentence compared to words in a word list in occipito-temporal regions reflects facilitation and/or prediction from the sentence context (activity in higher cortical regions) to specific word forms in occipitotemporal regions (e.g., the visual word form area).

\section{Alpha and beta oscillations in sentence processing}

\section{Word analysis}

We observed a similar spatial topography and time course in the alpha and beta bands-a stronger desynchronization in left-lateralized temporal, parietal, and frontal areas for words in a sentence context compared to words embedded in a word list. For both frequency bands, in temporo-parietal regions, the difference begins around $150 \mathrm{~ms}$, and is strongest around $350 \mathrm{~ms}$, while in left frontal regions, the difference begins around $150 \mathrm{~ms}$, and becomes stronger over time. The similarity between these two frequency bands is suggestive of a broadband desynchronization. A decrease in alpha/beta power is typically interpreted to reflect more (an increase in) activation of the underlying neuronal population, while a power increase reflects less (a decrease) in activation (Bastiaansen et al., 2008; Jensen and Mazaheri, 2010; Klimesch et al., 1997; Osipova et al., 2006). On this basis, the desynchronization effect indicates stronger neural activation for sentences than word lists. This effect could reflect unification of the incoming word in sentences (but not in word lists). The difference between sentences and word lists is in structure and sentence-level meaning (syntax and semantics), and not orthography, phonology, word meaning, or morphology because the same words were used in both conditions. Therefore, we are likely observing the unification of semantic and syntactic information. Importantly, the difference between late and early words in a sentence is not due to a difference in lexical frequency. We controlled for this using stratification methods.

A recent source-level MEG study observed a desynchronization between 8 and $30 \mathrm{~Hz}$ in bilateral occipital and parietal regions and left posterior temporal regions, following a semantic, or a syntactic violation (Kielar et al., 2015). In our study, reading a sentence also required processing of semantic and syntactic information, which could explain the similarity of the spatiotemporal distribution between our studies. The study of Kielar et al. thus supports our interpretation that semantic and syntactic unification involves the alpha and beta bands-by stronger recruitment of areas relevant for unification as indicated by the ERD in these frequency bands.

\section{Context analysis}

As the sentence unfolds the context imposes more constraints on what the upcoming word will be, which in turn should facilitate unification. Here, we observed two context effects in the alpha and beta bands.

First, a decrease in alpha and beta power (an increase in neural activation) for late words compared to early words in left frontal, temporal, and bilateral occipital regions. We interpret these findings as a context facilitatory effect, drawing inspiration from the MUC model (Hagoort, $2003,2005,2013)$. The model proposes that the lexical information of a word is represented in the activation of (predominantly) left temporal regions. The activation spreads to left frontal regions, which returns input to left temporal and parietal regions, and initiates a second wave of activation. Continuous cycles of activity sent between these regions build the context of the unfolding sentence. Applying this theory to our findings, the decrease in alpha and beta power as the sentence unfolds reflects the build-up of a context in left temporal regions, which spreads to left frontal regions to facilitate unification, and bilateral occipital regions to boost the activation (i.e. a power decrease) for recognizing certain words (orthographic properties). Evidence of interaction between context (semantics) and word recognition is found in several studies (e.g., Kim and Lai, 2012; Yap et al., 2012).

The second effect was in bilateral (but clearly right dominant) frontal and right parietal regions-an alpha and beta band power increase for late words compared to early words. These right-lateralized effects are absent from the context analysis for word lists, which suggests that the right-lateralized effects in sentences are due to a change in 
context (which is not present in word lists). The word analysis is a highlevel linguistic contrast (sentence vs. word list), and a lack of effect in this contrast in the right frontal and parietal regions further suggests that the consequence of a differential context between late and early words does not strictly involve linguistic processes, but might be due to a domain general, cognitive control process. On this basis, we speculate that the right-lateralized frontal-parietal regions reflect a network of regions that are less involved (hence more power) in unification late in a sentence when the context has a stronger facilitatory effect on unification.

In support of our interpretation, the spatial topography of our results is similar to the frontal-parietal network associated with domain general cognitive demands, which is referred to as the cognitive control, executive control, or multiple demand network (Corbetta and Shulman, 2002; Duncan, 2010; Fedorenko et al., 2013; Niendam et al., 2012). For example, Fedorenko et al., demonstrated at the single subject- and group-level that this network is activated when comparing difficult and easy versions of a task, independent of task type (non words vs. sentences, and simple vs. difficult math, working memory, and interference tasks). Previous studies have often found this frontal-parietal network to be bilateral (e.g., Duncan, 2010; Fedorenko et al., 2013). However, in certain tasks attentional processes have been demonstrated to be present in language tasks, in the form of a right-lateralized fronto-parietal network (Cristescu et al., 2006; Kristensen et al., 2012). On this basis, we propose that with these effects we tapped into an attentional network that directs more resources to the early than to the late words in a sentence, since early on in a sentence the context is weaker than towards the end of a sentence.

\section{Gamma oscillations in sentence processing}

In the word analysis, we observed an ERS for sentences compared to word lists at low and high gamma frequencies (Fig. 1). Low gamma effects were in left occipital, left parietal, left motor, and left temporal regions, while high gamma effects were in left frontal and left temporal regions. Interestingly, we observed low gamma effects to be earlier and located more posterior than late gamma effects (Fig. 1, right panel).

In a sentence, compared to a word list, the words form a meaningful and structured context which can be used to predict the next upcoming word. A plausible interpretation for the gamma ERS is that it reflects a correct prediction, i.e. a match between the predicted and observed word. As a correct prediction is only possible for semantic and syntactically well-formed sentences, this interpretation can explain the reduced lower gamma ERS, and the lack of high gamma ERS observed in word lists (Fig. 1, right panel). Our interpretation stems from Herrmann et al. (2004) who proposed a model that explains gamma responses (ERS) in terms of a match between bottom-up and top-down information. The sentences used in this study contained neither strong ambiguities nor grammatical violations, and thus allowed for preceding words to be used to predict the upcoming words-to narrow down the possibilities of various linguistic aspects of the upcoming word, such as, for instance, animacy, word category, and tense. Further support for our interpretation comes from a study that explicitly showed a gamma ERS $(40-50 \mathrm{~Hz})$ in left temporal and central sensors that was associated with words that had a high cloze probability given the preceding context (sentence), but not with words that could grammatically combine with the preceding sentence context and had a low cloze probability (Wang et al., 2012). Based on the above interpretation, we would expect an effect in the context analysis for sentences-as the context becomes stronger for late words, the match between the prediction and actual word has a higher probability of being correct. A similar effect is also expected based on previous studies that showed an increase in gamma power across grammatically correct sentences (albeit with EEG; Bastiaansen and Hagoort, 2015; Hald et al., 2006; Rommers et al., 2013). In our study, we did observe a higher gamma power for late than early words in sentences, but this was also the case in word lists (Fig. 1).

\section{Distinguishing oscillatory activity from evoked responses}

One general concern related to the interpretation of modulations in oscillatory activity is that the reported differences could reflect differences in the spectral representation of the event-related activity, which may not be strictly oscillatory in nature. This interpretational limit is particularly acute when the stimulus protocol leads to transients in the signal that are time-locked to the onset of the stimuli, as was unavoidable in our study. Since the transient event-related signal components typically contribute signal power in the frequency range up to about $20 \mathrm{~Hz}$, the above concern applies predominantly to the interpretation of frequency components up to the beta range. To address this concern, we performed a set of control analyses, in which we computed our contrasts of interest (for the theta, alpha, and beta ranges) for the event-related average of the band-limited signals, and for the power estimated after subtraction of the event-related average. The results of these analyses are shown in supplementary Fig. S3 (and a description is provided in the supplementary materials), and provide confirmatory evidence that the large majority of the results reported can be interpreted in terms of modulations in oscillatory activity. One exception may be the context effect for the theta band in left temporal regions, which shows a similar topography in the power and ERF contrasts (Fig. S3A). Yet, that does not exclude that we observed a modulation in a band-limited (centered at $5 \mathrm{~Hz}$ ) phase-locked oscillatory component, related to memory retrieval processes.

\section{Roles of left and right hemisphere in sentence processing}

Reviews of language studies demonstrate coordination and interaction between multiple brain areas during language processing (Fedorenko and Thompson-Schill, 2014; Price, 2010; Vartiainen et al., 2009; Halgren et al., 2002). The extent to which specific regions are activated appears to be task dependent. High-level core language tasks, such as sentence understanding, activate the dominant (left) hemisphere (Snijders et al., 2009; Xiang et al., 2010), whereas low-level tasks (e.g., orthographic and word sound analysis), and language tasks that involve non-language-specific components (e.g., attention, memory) have been shown to recruit bilateral fronto-parietal regions (Fedorenko and Thompson-Schill, 2014; Bozic et al., 2010). Our results are in agreement with this distinction. The word analysis aimed to tap aspects of combinatoriality in language (unification), and produced left hemisphere dominant results. In addition, the context analysis reflected changes in cognitive demands whereby effects were found in bilateral (but right hemisphere dominant) frontal, temporal, and parietal regions.

\section{Conclusion}

To summarize, we investigated the spatial and temporal dynamics of neural oscillations during sentence reading. We quantified the response to individual words in a sentence compared to a word list to determine the effects of context. In sentences, we observed changes in oscillatory power at the theta, alpha, beta, and gamma frequencies. We also quantified differences between words early versus later in a sentence to observe the changes in individual words as the context strengthened. We observed changes in left and right hemispheres in the theta, alpha, and gamma bands.

To the best of our knowledge, this is the first MEG source-localized sentence processing study that investigated how individual (grammatical) words are processed, and how this is influenced by an unfolding sentence context. Moreover, with 102 participants, this is the largest, most powered MEG study of its kind. Source-localization allowed us to conclude that oscillations localize to similar brain regions as those 
found in the fMRI literature: first, sentence (language) processing recruits a widely distributed network (Fedorenko and Thompson-Schill, 2014; Friederici and Singer, 2015; Hagoort and Indefrey, 2014). Second, the presence of effects in left temporal and left frontal regions suggests that, within this widely distributed network, these two regions are important in sentence processing. This is also in agreement with the MUC model of sentence processing (Hagoort, 2003, 2005, 2013). Third, involvement of the right frontal-parietal regions in sentence processing likely reflects engagement of the domain general cognitive control network according to task demands at different points in a sentence.

In this study, we also made use of the temporal resolution in MEG. We demonstrated that unification processes involved in reading a sentence begin around $200 \mathrm{~ms}$ when statistically comparing sentences with word lists. Moreover, visual inspection of the oscillatory power time courses show that differentiation between the wave forms for sentences and word lists occurs around $100 \mathrm{~ms}$, which suggests that the semantic and syntactic context effects can begin as early as $100 \mathrm{~ms}$ (see Fig. 1, e.g., in the left frontal region in the beta band, and left occipito-parietal region in the lower gamma band). The early onset of language effects following the language stimuli is in accordance with the abundance of MEG and EEG studies reporting language effects within the first $400 \mathrm{~ms}$ following word onset (see Salmelin, 2007 for a review of MEG language studies at the single word level and beyond).

We analyzed multiple frequencies and found that the theta, alpha, beta, and gamma bands are all involved in sentence processing. This highlights the importance of studying multiple frequencies to provide a broader perspective on which oscillations are involved and which are not involved in any given cognitive task. The observation of oscillatory changes in multiple brain regions at several frequency bands suggests no simple mapping between a specific region and function. Almost certainly, the effects in the different frequency bands have different functional significance. The presence of effects in the frequency bands that we observed indicate that higher order language processing (i.e. unification) depends on multiple networks, including memory networks in temporal cortex and attentional networks in the right parieto-frontal network, in addition to the core language network in left perisylvian cortex. The abundant effects in occipital areas, moreover, imply that higher order language processes interact with the extraction of relevant information from the feedforward visual processing stream. The timing of all these effects are a testimony of the exquisite speed at which the human brain is able to complete the complex cascade of processes that are involved in extracting meaning from a series of orthographic scribbles entering primary visual cortex.

\section{Acknowledgments}

We thank Laura Arendsen, Manuela Schuetze, Tineke de Haan, and Charlotte Poulisse for participant recruitment, stimuli construction, and coding. We further thank Laura Arendsen and Manuela Schuetze for assisting with data preprocessing. This research was supported by the International Max Planck Research School for Language Science (for N.H.L.L), and the Spinoza Prize Award and the Language in Interaction Gravitation grant from the Netherlands Organization for Scientific Research (for P.H.).

Data were collected as part of the MOUS (Mother of all Unification Studies) project. In due time, the MOUS data will be made available to the wider research community.

\section{Appendix A. Supplementary data}

Supplementary data to this article can be found online at http://dx. doi.org/10.1016/j.neuroimage.2016.03.007.

\section{References}

Bastiaansen, M., Hagoort, P., 2015. Frequency-based segregation of syntactic and semantic unification during online sentence level language comprehension. J. Cogn. Neurosci. 1-13. http://dx.doi.org/10.1162/jocn.

Bastiaansen, M., van Berkum, J., Hagoort, P., 2002. Syntactic processing modulates the $\theta$ rhythm of the human EEG. NeuroImage 17 (3), 1479-1492. http://dx.doi.org/10. 1006/nimg.2002.1275.

Bastiaansen, M., van der Linden, M., Ter Keurs, M., Dijkstra, T., Hagoort, P., 2005. Theta responses are involved in lexical-semantic retrieval during language processing. J. Cogn. Neurosci. 17 (3), 530-541. http://dx.doi.org/10.1162/0898929053279469.

Bastiaansen, M. Oostenveld, R., Jensen, O., Hagoort P. 2008. I see what you mean: theta power increases are involved in the retrieval of lexical semantic information. Brain Lang. 106 (1), 15-28. http://dx.doi.org/10.1016/j.bandl.2007.10.006.

Bastiaansen, M., Magyari, L., Hagoort, P., 2010. Syntactic unification operations are reflected in oscillatory dynamics during on-line sentence comprehension. J. Cogn. Neurosci. 22 (7), 1333-1347.

Bozic, M., Tyler, L.K., Ives, D.T., Randall, B., Marslen-wilson, W.D., 2010. Bihemispheric foundations for human speech comprehension. Proc. Natl. Acad. Sci. 107 (40), 17439-17444. http://dx.doi.org/10.1073/pnas.1000531107.

Cashdollar, N., Malecki, U., Rugg-gunn, F.J., Duncan, J.S., Lavie, N., Duzel, E., 2009. Hippocampus-dependent and -independent theta-networks of active maintenance. Proc. Natl. Acad. Sci. 106 (48), 20493-20498.

Chein, J.M., Moore, A.B., Conway, A.R.a., 2011. Domain-general mechanisms of complex working memory span. NeuroImage 54 (1), 550-559. http://dx.doi.org/10.1016/j. neuroimage.2010.07.067.

Corbetta, M., Shulman, G.L., 2002. Control of goal-directed and stimulus-driven attention in the brain. Nat. Rev. Neurosci. 3 (3), 201-215. http://dx.doi.org/10.1038/nrn755.

Cristescu, T.C., Devlin, J.T., Nobre, A.C., 2006. Orienting attention to semantic categories. Neurolmage 33 (4), 1178-1187. http://dx.doi.org/10.1016/j.neuroimage.2006.08. 017.

Crone, N.E., Miglioretti, D.L., Gordon, B., Lesser, R.P., 1998. Functional mapping of human sensorimotor cortex with electrocorticographic spectral analysis II. Event-related synchronization in the gamma band. Brain 121, 2301-2315.

Dalal, S.S., Guggisberg, A.G., Edwards, E., Sekihara, K., Findlay, A.M., Canolty, R.T., ... Nagarajan, S.S., 2008. Five-dimensional neuroimaging: localization of the timefrequency dynamics of cortical activity. NeuroImage 40 (4), 1686-1700. http://dx. doi.org/10.1016/j.neuroimage.2008.01.023.

Dale, A.M., Fischl, B., Sereno, M.I., 1999. Cortical surface-based analysis. NeuroImage 194, 179-194. http://dx.doi.org/10.1006/nimg.1998.0395.

Davidson, D.J., Indefrey, P., 2007. An inverse relation between event-related and timefrequency violation responses in sentence processing. Brain Res. 1158, 81-92. http://dx.doi.org/10.1016/j.brainres.2007.04.082.

Dehaene, S., Le Clec'H, G., Poline, J.-B., Le Bihan, D., Cohen, L., 2002. The visual word form area: a prelexical representation of visual words in the fusiform gyrus. Neuroreport 13 (3), 321-325. http://dx.doi.org/10.1097/00001756-200203040-00015.

Deiber, M., Missonnier, P., Bertrand, O., Gold, G., Fazio-costa, L., Iban, V., Giannakopoulos, P., 2007. Distinction between perceptual and attentional processing in working memory tasks: a study of phase-locked and induced oscillatory brain dynamics. J. Cogn. Neurosci. 19 (1), 158-172.

Duncan, J., 2010. The multiple-demand (MD) system of the primate brain: mental programs for intelligent behaviour. Trends Cogn. Sci. 14 (4), 172-179. http://dx.doi. org/10.1016/j.tics.2010.01.004.

Engel, A.K., Fries, P., Singer, W., 2001. Dynamic predictions: oscillations and synchrony in top-down processing. Nat. Rev. Neurosci. 2 (October), 704-716.

Fedorenko, E., Thompson-Schill, S.L., 2014. Reworking the language network. Trends Cogn. Sci. 18 (3), 120-126. http://dx.doi.org/10.1016/j.tics.2013.12.006.

Fedorenko, E, Duncan, J., Kanwisher, N , 2013. Broad domain generality in focal regions of frontal and parietal cortex. Proc. Natl. Acad. Sci. http://dx.doi.org/10.1073/pnas. 1315235110 (/-/DCSupplemental.www.pnas.org/cgi/doi/10.1073/pnas.1315235110).

Friederici, A.D., Singer, W., 2015. Grounding language processing on basic neurophysiological principles. Trends Cogn. Sci. 1-10. http://dx.doi.org/10.1016/j.tics.2015.03.012.

Gross, J., Kujala, J., Hämäläinen, M., Timmermann, L., Schnitzler, A., Salmelin, R., 2001. Dynamic imaging of coherent sources: studying neural interactions in the human brain. Proc. Natl. Acad. Sci. 98 (2), 694-699.

Guderian, S., Düzel, E., 2005. Induced theta oscillations mediate large-scale synchrony with mediotemporal areas during recollection in humans. Hippocampus 15 (7), 901-912. http://dx.doi.org/10.1002/hipo.20125.

Hagoort, P., 2003. How the brain solves the binding problem for language: a neurocomputational model of syntactic processing. NeuroImage 20, 18-29. http:// dx.doi.org/10.1016/j.neuroimage.2003.1796.

Hagoort, P., 2005. On Broca, brain, and binding: a new framework. Trends Cogn. Sci. 9 (9), 416-423. http://dx.doi.org/10.1016/j.tics.2005.07.004.

Hagoort, P., 2013. MUC (memory, unification, control) and beyond. Front. Psychol. 4 (July), 416. http://dx.doi.org/10.3389/fpsyg.2013.00416.

Hagoort, P., Indefrey, P., 2014. The neurobiology of language beyond single words. Annu. Rev. Neurosci. 37, 347-362. http://dx.doi.org/10.1146/annurev-neuro071013-013847.

Hagoort, P., Poeppel, D., 2013. The Infrastructure of the Language-Ready Brain. In: Arbib, M.A. (Ed.), Language, Music, and the Brain: A Mysterious Relationships. MIT Press, Cambridge, MA, pp. 233-255.

Hald, L., Bastiaansen, M., Hagoort, P., 2006. EEG theta and gamma responses to semantic violations in online sentence processing. Brain Lang. 96 (1), 90-105. http://dx.doi. org/10.1016/j.bandl.2005.06.007.

Halgren, E., Dhond, R.P., Christensen, N., Van Petten, C., Marinkovic, K., Lewine, J.D., Dale, A.M., 2002. N400-like Magnetoencephalography Responses Modulated by Semantic 
Context, Word Frequency, and Lexical Class in Sentences. Neurolmage 17 (3), 1101-1116. http://dx.doi.org/10.1006/nimg.2002.1268.

Hari, R., Salmelin, R., 2012. Magnetoencephalography: from SQUIDs to neuroscience. NeuroImage 61 (2), 386-396. http://dx.doi.org/10.1016/j.neuroimage.2011.11.074.

Hauck, M., Lorenz, J., Engel, A.K., 2007. Attention to painful stimulation enhances gammaband activity and synchronization in human sensorimotor cortex. J. Neurosci. 27 (35), 9270-9277. http://dx.doi.org/10.1523/JNEUROSCI.2283-07.2007.

Herrmann, C.S., Munk, M.H.J.. Engel, A.K., 2004. Cognitive functions of gamma-band activity: memory match and utilization. Trends Cogn. Sci. 8 (8), 347-355. http://dx.doi.org/10. 1016/j.tics.2004.06.006.

Jensen, O., Mazaheri, A., 2010. Shaping functional architecture by oscillatory alpha activity: gating by inhibition. Front. Hum. Neurosci. 4, 186. http://dx.doi.org/10.3389/ fnhum.2010.00186.

Jensen, O., Tesche, C.D., 2002. Frontal theta activity in humans increases with memory load in a working memory task. Eur. J. Neurosci. 15, 1395-1399.

Keuleers, E., Brysbaert, M., New, B., 2010. SUBTLEX-NL: a new measure for Dutch word frequency based on film subtitles. Behav. Res. Methods 42 (3), 643-650. http://dx. doi.org/10.3758/BRM.42.3.643.

Kielar, A., Panamsky, L., Links, Kira A., Meltzer, Jed A., 2015. Localization of electrophysiological responses to semantic and syntactic anomalies in language comprehension with MEG. Neurolmage 105, 507-524. http://dx.doi.org/10.1016/j.neuroimage.2014. 11.016.

Kim, A., Lai, V., 2012. Rapid interactions between lexical semantic and word form analysis during word recognition in context: evidence from ERPs. J. Cogn. Neurosci. 24 (5), 1104-1112. http://dx.doi.org/10.1162/jocn_a_00148.

Klimesch, W., Doppelmayr, M., Pachinger, T., Russegger, H., 1997. Event-related desynchronization in the alpha band and the processing of semantic information. Cogn. Brain Res. 6 (2), 83-94. http://dx.doi.org/10.1016/S0926-6410(97)00018-9.

Klimesch, W., Doppelmayr, M., Stadler, W., Po, D., Sauseng, P., Ro, D., 2001. Episodic retrieval is reflected by a process specific increase in human electroencephalographic theta activity. Neurosci. Lett. 302, 49-52.

Klimesch, W., Freunberger, R., Sauseng, P., Gruber, W., 2008. A short review of slow phase synchronization and memory: evidence for control processes in different memory systems? Brain Res. 1235, 31-44. http://dx.doi.org/10.1016/j.brainres.2008.06.049.

Klimesch, W., Freunberger, R., Sauseng, P., 2010. Oscillatory mechanisms of process binding in memory. Neurosci. Biobehav. Rev. 34 (7), 1002-1014. http://dx.doi.org/10. 1016/j.neubiorev.2009.10.004.

Kristensen, L.B., Wang, L., Petersson, K.M., Hagoort, P., 2012. The interface between language and attention: prosodic focus marking recruits a general attention network in spoken language comprehension. Cereb. Cortex 23 (8), 1836-1848. http://dx.doi. org/10.1093/cercor/bhs164.

Marcus, D.S., Harwell, J., Olsen, T., Hodge, M., Glasser, M.F., Prior, F., ... Van Essen, D.C., 2011. Informatics and data mining tools and strategies for the Human Connectome project. Front. Neuroinform. 5 (June), 1-12. http://dx.doi.org/10.3389/fninf.2011.00004.

Maris, E., Oostenveld, R., 2007. Nonparametric statistical testing of EEG- and MEG-data. J. Neurosci. Methods 164 (1), 177-190. http://dx.doi.org/10.1016/j.jneumeth.2007. 03.024 .

Mitra, P.P., Pesaran, B., 1999. Analysis of dynamic brain imaging data. Biophys. J. 76 (February), 691-708.

Mormann, F., Fell, J., Axmacher, N., Weber, B., Lehnertz, K., Elger, C.E., Fernández, G., 2005. Phase/amplitude reset and theta-gamma interaction in the human medial temporal lobe during a continuous word recognition memory task. Hippocampus 15 (7), 890-900. http://dx.doi.org/10.1002/hipo.20117.

Mummery, C.J., Patterson, K., Price, C.J., Ashburner, J., Frackowiak, R.S.J., Hodges, J.R., 2000. A voxel-based morphometry study of semantic dementia: relationship between temporal lobe atrophy and semantic memory. Ann. Neurol. 47 (1), 36-45. http://dx.doi. org/10.1002/1531-8249(200001)47:1<36::AID-ANA8>3.0.CO;2-L.

Niendam, T.A., Laird, A.R., Ray, K.L., Dean, Y.M., Glahn, D.C., Carter, C.S., 2012. Metaanalytic evidence for a superordinate cognitive control network subserving diverse executive functions. Cogn. Affect. Behav. Neurosci. 12 (2), 241-268. http://dx.doi. org/10.3758/s13415-011-0083-5.
Nolte, G., 2003. The magnetic lead field theorem in the quasi-static approximation and its use for magnetoencephalography forward calculation in realistic volume conductors. Phys. Med. Biol. 48 (22), 3637-3652. http://dx.doi.org/10.1088/0031-9155/48/ 22/002.

Oostenveld, R., Fries, P., Maris, E., Schoffelen, J.M., 2011. FieldTrip: open source software for advanced analysis of MEG, EEG, and invasive electrophysiological data. Comput. Intell. Neurosci. 2011, 156869. http://dx.doi.org/10.1155/2011/156869.

Osipova, D., Takashima, A., Oostenveld, R., Fernandez, G., Maris, E., Jensen, O., 2006. Theta and gamma oscillations predict encoding and retrieval of declarative memory. J. Neurosci. 26 (28), 7523-7531. http://dx.doi.org/10.1523/JNEUROSCI.1948-06.2006.

Patterson, K., Nestor, P.J., Rogers, T.T., 2007. Where do you know what you know? the representation of semantic knowledge in the human brain. Nat. Rev. Neurosci. 8 (12), 976-987. http://dx.doi.org/10.1038/nrn2277.

Pfurtscheller, G., 1992. Event-related synchronization (ERS): an electrophysiological correlate of cortical areas at rest. Electroencephalogr. Clin. Neurophysiol. 83 (1), 62-69. http://dx.doi.org/10.1016/0013-4694(92)90133-3.

Pfurtscheller, G., Aranibar, A., 1977. Event-related cortical desynchronization detected by power measurements of scalp EEG. Electroencephalogr. Clin. Neurophysiol. 42 (6), 817-826. http://dx.doi.org/10.1016/0013-4694(77)90235-8.

Price, C.J., 2010. The anatomy of language: a review of $100 \mathrm{fMRI}$ studies published in 2009. Ann. N. Y. Acad. Sci. 62-88 http://dx.doi.org/10.1111/j.1749-6632.2010.05444.x.

Rommers, J., Dijkstra, T., Bastiaansen, M., 2013. Context-dependent semantic processing in the human brain: evidence from idiom comprehension. J. Cogn. Neurosci. 25 (5), 762-776. http://dx.doi.org/10.1162/jocn_a_00337.

Salmelin, R., 2007. Clinical neurophysiology of language: the MEG approach. Clin. Neurophysiol. 118 (2), 237-254. http://dx.doi.org/10.1016/j.clinph.2006.07.316.

Särelä, J., Valpola, H., 2005. Denoising source separation. J. Mach. Learn. Res. 6 (3), 233-272.

Schoffelen, J.M., Oostenveld, R., Fries, P., 2005. Neuronal coherence as a mechanism of effective corticospinal interaction. Science 308, 111-113. http://dx.doi.org/10.1126/ science. 1107027.

Silvanto, J., Muggleton, N., Lavie, N., Walsh, V., 2009. The perceptual and functional consequences of parietal top-down modulation on the visual cortex. Cereb. Cortex 19, 327-330. http://dx.doi.org/10.1093/cercor/bhn091.

Snijders, T.M., Vosse, T., Kempen, G., Berkum, J.A.Van, Petersson, K.M., Hagoort, P., 2009 Retrieval and Unification of Syntactic Structure in Sentence Comprehension : an fMRI Study Using Word-Category Ambiguity, (July). pp. 1493-1503 http://dx.doi. org/10.1093/cercor/bhn187.

Stolk, A., Todorovic, A., Schoffelen, J.M., Oostenveld, R., 2013. Online and offline tools for head movement compensation in MEG. Neurolmage 68, 39-48. http://dx.doi.org/ 10.1016/j.neuroimage.2012.11.047.

Tarkiainen, A., Helenius, P., Hansen, P.C., Cornelissen, P.L., Salmelin, R., 1999. Dynamics of letter string perception in the human occipitotemporal cortex. Brain 122, 2119-2131.

Taylor, P.C.J., Nobre, A.C., Rushworth, M.F.S., 2007. Subsecond changes in top down contro exerted by human medial frontal cortex during conflict and action selection: a combined transcranial magnetic stimulation electroencephalography study. J. Neurosci. 27 (42), 11343-11353. http://dx.doi.org/10.1523/JNEUROSCI.2877-07.2007.

Vartiainen, J., Parviainen, T., Salmelin, R., 2009. Spatiotemporal convergence of semantic processing in reading and speech perception. J. Neurosci. 29 (29), 9271-9280 http://dx.doi.org/10.1523/JNEUROSCI.5860-08.2009.

Wang, L., Zhu, Z., Bastiaansen, M., 2012. Integration or predictability? A further specification of the functional role of gamma oscillations in language comprehension. Front Psychol. 3, 1-12. http://dx.doi.org/10.3389/fpsyg.2012.00187.

Xiang, H.D., Fonteijn, H.M., Norris, D.G., Hagoort, P., 2010. Topographical functional connectivity pattern in the perisylvian language networks. Cereb. Cortex (New York, N.Y. : 1991) 20 (3), 549-560. http://dx.doi.org/10.1093/cercor/bhp119.

Yap, M.., Pexman, P.M. Wellsby, M., Hargreaves, I.S., Huff, M.J., 2012. An abundance of riches: cross-task comparisons of semantic richness effects in visual word recognition. Front. Hum. Neurosci. 6, 72. http://dx.doi.org/10.3389/fnhum.2012.00072. 